\title{
Recurrent Strokes due to Transient Vasospasms of the Extracranial Internal Carotid Artery
}

\author{
Sigrid Wöpking ${ }^{a}$ Andreas Kastrup $^{a} \quad$ Markus Lentschig ${ }^{b}$ \\ Freimuth Brunner ${ }^{\mathrm{a}}$ \\ ${ }^{a}$ Department of Neurology, Klinikum Bremen-Mitte, and ${ }^{b} Z E M O D I$, Bremen, Germany
}

\section{Key Words}

Vasospasm · Internal carotid artery · Cerebral ischemia · Duplex sonography · Magnetic resonance angiography

\begin{abstract}
Vasospasms of the intracranial arteries are a well-known complication of subarachnoid hemorrhage and are also frequently encountered in other disorders such as migraine, cerebral vasculitis or reversible cerebral vasoconstriction syndrome. In contrast, recurrent spontaneous vasospasms of the extracranial circulation appear to be extremely rare and have most often been associated with migraine. We present a patient with recurrent strokes due to spontaneous transient vasospastic occlusions of the internal carotid artery (ICA) without migraine over a time period of at least 13 years. Initially, the patient had presented with a bilateral ICA occlusion and a cerebral infarct on the right side. While the right ICA remained occluded, a reopening of the left ICA could be detected 3 days after this initial event. In subsequent years, both duplex sonography and magnetic resonance angiography revealed recurrent occlusions of the left ICA, which resolved spontaneously within days. This case and other rare previous reports indicate that recurrent non-migrainous vasospasms of the extracranial carotid artery likely reflect a distinct entity which can cause ischemic strokes.
\end{abstract}

(c) 2013 S. Karger AG, Basel

\section{Introduction}

Vasospasms of the intracranial arteries are a common and well-known complication of subarachnoid hemorrhage [1]. They are also encountered in other disorders such as cerebral

Andreas Kastrup, MD

Department of Neurology, Klinikum Bremen-Mitte

St.-Jürgen-Strasse 1

DE-28177 Bremen (Germany)

E-Mail akastru@gwdg.de 
Wöpking et al.: Recurrent Strokes due to Transient Vasospasms of the Extracranial Internal Carotid Artery

vasculitis or reversible cerebral vasoconstriction syndrome (RCVS) [2]. In contrast, vasospasms of the extracranial circulation occur less frequently and have most often been associated with mechanical manipulations [3] or ergot poisoning [4]. Recurrent spontaneous vasospasms occurring mainly within the intracranial but also within the extracranial arteries have also been reported in patients suffering from migraine headaches [5].

We present a patient with recurrent strokes due to transient vasospastic occlusions of the internal carotid artery (ICA) without migrainous headaches. Our case supports the notion that recurrent non-migrainous vasospasms of the extracranial carotid artery likely reflect a distinct entity which can cause ischemic strokes.

\section{Case Report}

In July 1999, a previously healthy 34-year-old female patient was admitted to our hospital for the first time with an acute left-sided brachiofacial hemiparesis. She reported that similar symptoms had occurred several times in the previous 10 years but had regressed spontaneously. Aside from regular nicotine consumption, she had no other cardiovascular risk factors. In addition, she had no history of migraine headaches.

Magnetic resonance imaging (MRI) showed an internal middle cerebral artery (MCA) territory watershed infarct, probably of hemodynamic origin. Duplex sonography on admission revealed proximal occlusions of both ICAs and retrograde flow in both supratrochlear arteries (STA). Three days later, a digital subtraction angiography showed a recanalization of the left ICA and a persistent occlusion of the right ICA at the origin, which subsequently never recanalized. On axial T1-weighted fat-suppressed images, the presence of intramural hematomas and thus arterial dissections as a cause of the bilateral ICA occlusions could be ruled out. A cerebrospinal fluid analysis was unremarkable.

Under the assumption of vascular spasms, the patient was treated with the calcium antagonist nimodipine $(240 \mathrm{mg} /$ day) and was heparinized with an aim for a partial thromboplastin time of $>60 \mathrm{~s}$. Despite this treatment, a transient occlusion of the left ICA, confirmed with repeated ultrasound and magnetic resonance angiography (MRA), occurred again during this first admission. The patient was discharged receiving nimodipine, and an oral anticoagulation with phenprocoumon was begun.

In February 2004, she was readmitted due to a short episode with paresis of the right hand and aphasia. While MRI including diffusion-weighted imaging showed no new infarction, an initial occlusion and subsequent reopening of the left ICA was documented with ultrasound and MRA. During the course of this admission, oral anticoagulation was discontinued and the patient was discharged with Aspirin and nimodipine.

In March 2010, February 2011 and May 2011, the patient experienced recurrent episodes with transient right-sided hemiparesis and aphasia. In all three instances, MRI revealed new small infarcts in the left MCA territory. Extensive laboratory investigations were performed twice (including virology, bacteriology and tests for thrombophilia and vasculitis), but revealed no pathological results. Likewise, 2 chest X-rays and an ultrasound of the abdomen were normal.

Similar to the findings in 2004, MRA and ultrasound repeatedly revealed an initial occlusion and subsequent reopening of the left ICA (fig. 1, fig. 2) without nimodipine administration. During the episodes of left ICA occlusion, a reduced pulsatility in the left MCA, a collateralization via the posterior communicating arteries and a retrograde flow within the ipsilateral STA were observed with ultrasound. After recanalization, ultrasound showed an antegrade flow within the ipsilateral STA and a completely normalized MCA flow. 
Wöpking et al.: Recurrent Strokes due to Transient Vasospasms of the Extracranial Internal Carotid Artery

Since May 2011, the patient has had no further ischemic symptoms. A coincidence with smoking was presumed during the first stays, but attacks appeared also after she quit smoking.

\section{Discussion}

Evidence is accumulating that recurrent vasospasms of the extracranial ICA are a rare but important cause of ischemic disorders mainly in young patients [6-14]. While previous cases have been associated with migraine $[5,10]$, we describe a patient with recurrent nonmigrainous vasospasms of the extracranial ICA. Only a few such cases have been reported in the literature to date $[6,8,11-14]$. It is noteworthy that the recurrent migrainous and nonmigrainous vasospasms of the extracranial ICA vasospasms appear to occur exclusively in the intermediate portion of the ICA $[8,11,13,14]$. The reason for this finding is currently unclear, but could be associated with a distinct pattern of sympathetic vasomotor innervation in the intermediate ICA segment, enhancing the susceptibility of this vessel segment to sympathetic stimuli among others [13]. It should also be emphasized that recurrent vasospasms of the extracranial ICA can only be observed during a short time period, and, therefore, they can only be detected using serial ultrasound or other vessel imaging modalities. It is thus likely that the incidence of this entity is underestimated.

In young patients with an acute ischemic stroke due to an ICA occlusion, the most important differential diagnosis is a dissection of the carotid artery. In light of a missing wall hematoma on repeated T1-weighted fat-suppressed images, as well as the recurrence of the episodes with spontaneous resolution of the left ICA occlusion within a few days, it is very unlikely that repeated dissections were responsible for the clinical course in our patient. The rapid spontaneous resolution of the ICA occlusion is also an argument against the presence of an inflammatory vessel disease. Although the beaded appearance of the left ICA could principally be suggestive of vasculitis, extensive laboratory evaluations were performed to rule out the presence of such a disorder. In particular, there was no evidence of Wegener's disease due to missing oropharyngeal inflammation, chest X-rays were inconspicuous and cand p-ANCA were negative.

Reversible vessel occlusions can also be observed in the context of RCVS. In the past few years, this disorder has emerged as a distinct entity and is characterized by thunderclap headaches accompanied by multifocal cerebral artery vasoconstriction. For the diagnosis of RCVS, a complete reversibility of the latter feature within 12 weeks of onset is required [2]. The missing headaches, as well as the time course argue against the presence of RCVS as the cause of the reversible ICA occlusions in our patient.

Currently, the optimal treatment of patients with recurrent vasospasms of the extracranial ICA remains unclear. In contrast to other case reports $[6,7,12]$ which reported positive effects of calcium channel inhibitors and corticoids, this treatment was not effective in our patient. In addition, neither oral anticoagulation nor platelet inhibitors prevented new stroke episodes in our case. Recently, Moeller et al. [13] demonstrated a sympathetic hypersensitivity in a patient with an isolated vasospasm of the extracranial ICA, which partially resolved using the $\alpha$-1-blocker prazosin. Therefore, an adrenoceptor blockade could become an interesting treatment option in these patients. 
Wöpking et al.: Recurrent Strokes due to Transient Vasospasms of the Extracranial Internal Carotid Artery

\section{Conclusion}

Spontaneous vasospasms of the extracranial ICA should be thought of in recurrent ischemic symptoms in young stroke patients. Recurrent non-migrainous vasospasms of the extracranial ICA likely reflect a distinct disease entity which can cause ischemic strokes. The etiology and the best medical treatment of this disorder still remain unclear.

\section{References}

1 Dorsch N: A clinical review of cerebral vasospasm and delayed ischaemia following aneurysm rupture. Acta Neurochir Suppl 2011;110:5-6.

2 Chen SP, Fuh JL, Wang SJ: Reversible cerebral vasoconstriction syndrome: current and future perspectives. Expert Rev Neurother 2011;11:1265-1276.

-3 Smith PG, Killeen TE: Carotid artery vasospasm complicating extensive skull base surgery: cause, prevention, and management. Otolaryngol Head Neck Surg 1987;97:1-7.

-4 Senter HJ, Lieverman AN, Pinto R: Cerebral manifestations of ergotism. Report of a case and review of the literature. Stroke 1976;7:88-92.

-5 Sanin LC, Mathew NT: Severe diffuse intracranial vasospasm as a cause of extensive migrainous cerebral infarction. Cephalalgia 1993;13:289-292.

6 Arning C, Schrattenholzer A, Lachenmayer L: Cervical carotid artery vasospasms causing cerebral ischemia: detection by immediate vascular ultrasonographic investigation. Stroke 1998;29:1063-1066.

7 Janzarik WG, Ringleb PA, Reinhard M, Rauer S: Recurrent extracranial carotid artery vasospasms: report of 2 cases. Stroke 2006;37:2170-2173.

8 Yoshimoto H, Matsuo S, Umemoto T, Kawakami N, Moriyama T: Idiopathic carotid and coronary vasospasm: a new syndrome? J Neuroimaging 2011;21:273-276.

-9 Solomon S, Lipton RB, Harris PY: Arterial stenosis in migraine: spasm or arteriopathy? Headache1990;30:52-61.

10 Magnin E, Mouton S, Abouaf L, Dumas-Stoeckel S, Hermier M, Tilikete C, Vighetto A: Idiopathic vasospastic angiopathy of the internal carotid arteries: a rarely recognized cause of ischemic stroke in young individuals. Rev Neurol (Paris) 2011;167:626-631.

$\checkmark 11$ Yokoyama H, Yoneda M, Abe M, Sakai T, Sagoh T, Adachi Y, Kondo T: Internal carotid artery vasospasm syndrome: demonstration by neuroimaging. J Neurol Neurosurg Psychiatry 2006;77:888-889.

$>12$ Kuzumoto Y, Mitsui Y, Ueda H, Kusunoki S: Vasospastic cerebral infarction induced by smoking: a case report. No To Shinkei 2005;57:33-36.

13 Moeller S, Hilz MJ, Blinzler C, Koehn J, Doerfler A, Schwab S, Koehrmann M: Extracranial internal carotid artery vasospasm due to sympathetic dysfunction. Neurology 2012;78:1892-1894.

14 Dembo T, Tanahashi N: Recurring extracranial internal carotid artery vasospasm detected by intravascular ultrasound. Intern Med 2012;51:1249-1253. 


\section{Case Reports in Neurology}

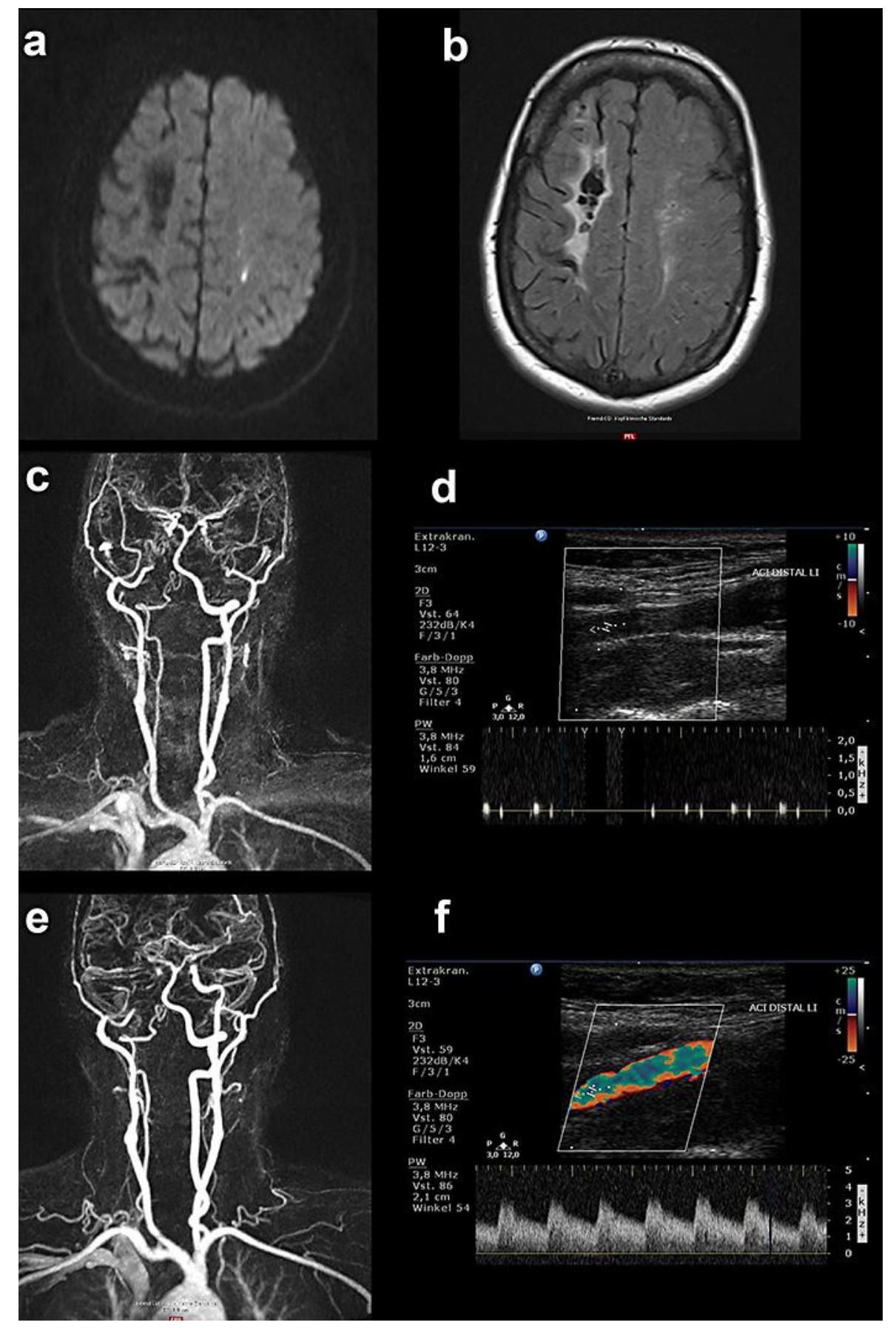

Fig. 1. a-f Magnetic resonance images obtained during admission in February 2011. a Diffusion-weighted image showing new internal MCA territory watershed infarctions in the left hemisphere. b FLAIR image showing the old watershed stroke in the territory of the right MCA, which occurred in 1999. c-f Contrastenhanced MRA and color-coded duplex showing an occlusion of the left ICA and subsequent complete recanalization within $48 \mathrm{~h}$.

\begin{tabular}{l|l}
\hline Case Rep Neurol 2013;5:143-148 & \\
\hline DOI: $10.1159 / 000354827$ & $\begin{array}{l}\text { ○ 2013 S. Karger AG, Basel } \\
\text { www.karger.com/crn }\end{array}$ \\
\hline
\end{tabular}

Wöpking et al.: Recurrent Strokes due to Transient Vasospasms of the Extracranial Internal Carotid Artery 


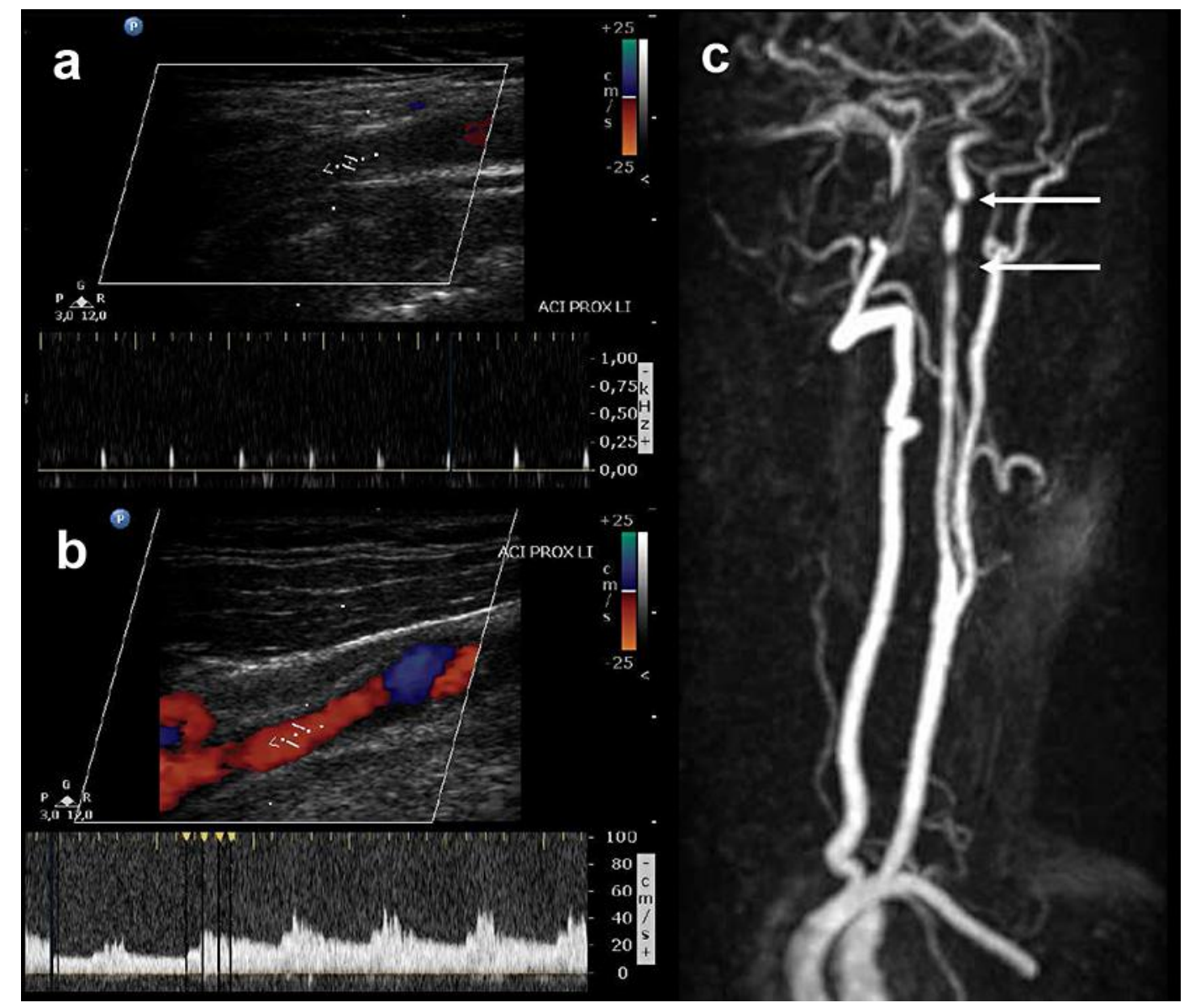

Fig. 2. Serial color-coded duplex obtained on the 29th and 30th of May 2011 showing a complete occlusion of the left ICA (a) and a subsequent incomplete recanalization with a dampened flow signal (b). Contrast-enhanced extracranial MRA (c) obtained on the 30th of May 2011 showing residual spastic vessel segments (arrows). 